A book from such a quarter might be expected to have as its aim the explanation of the physical processes that take place during, and constitute, creep deformation. However, knowledge has not reached the stage where this is fully possible. The most one can hope for is a statement and discussion of the present position, and this is what the book achieves. It does this in a non-mathematical way, on the whole, although some mathematics is used in the chapters on transient and steady-state creep.

The chapters are: (1) "Characteristics of the Creep Curve", which discusses the different types of curve and gives empirical formulæ ; (2) "Crystalline Flow", which describes one of the best-understood deformation processes and is accordingly one of the most specific chapters ; (3) "Metallographic Features of Creep", which deals principally with the surface manifestations of creep in aluminium; (4) "Grain Boundary Creep", largely devoted to a review of the work of T. S. $\mathrm{Ke}$ on small grain-boundary displacements; (5) "Transient Creep", which discusses the current theories of transient creep in the light of knowledge of actual creep curves; (6) "Steady State Creep", which is mainly a review and a critical discussion of theories that have been put forward and recounts the fow experiments that have been done with the aim of testing these theories; (7) "Tertiary Creep"; and (8) "The Development of Creep Resistant Alloys", which deals with the development during the past decade of alloys suitable for service at very high temperatures.

The information contained in the first seven chapters did not contribute much to this development compared with the help that a strong, coherent theory of creep could give. On the last page is given a programme of research to acquire the experimental material necessary to develop such a theory. One suspects that, if this is to be accomplished in a reasonable time, the proportion-probably small at present - of research workers engaged on this kind of work will have to be considerably increased.

Some misprints have been noticed: "eqn. 28" on p. 47 should be "eqn. 36" ; the term " $c$ " is omitted from eqn. 40 on p. 48 ; eqn. 54 on p. 55 should include the term " $Y 6$ " in place of " $Y S$ " to be consistent with eqn. 53. Sampling the index showed it to be fairly accurate ; the subject was at least always mentioned on the page indicated.

The book seems dear at $15 s$. for seventy-six pages. However, its quality is such that it will be used by most people having to deal with creep. It is suitable not only for physicists but will also be equally useful to metallurgists and engineers concerned with creep.

\section{ALGEBRAIC FUNCTIONS}

Introduction to the Theory of Algebraic Functions of One Variable

By Claude Chevalley. (Mathematical Surveys, No. 6.) Pp. xi+188. (New York: American Mathematical Society, 1951.) 4 dollars.

"A LL specialized research," says Schrödinger", "has real value only in the context of the integrated totality of knowledge." Prof. C. Chevalley's book is an account of specialized research which is so important that the review in the Bulletin of the American Mathematical Society ${ }^{2}$ extends to nearly fourteen pages. On the other hand, the subject is treated in a one-sided manner which may support the pessimist who feared that some day no two mathematicians will be able to understand each other. Weyl remarked that "the angel of topology and the devil of abstract algebra fight for the soul of each individual domain". Some consider that two words in this pronouncement should be interchanged, but no one can doubt that the second antagonist, however he may be described, has gained a complete victory in this book.

In his introduction, Prof. Chevalley gives a defence of his treatment. $\mathrm{He}$ is, of course, well aware that the theory of algebraic functions is closely allied to that of the algebraic geometry of curves. This alliance is so close that many consider the geometric treatment of algebraic functions to be the natural method. But there is a second method, that of abstract algebra, which emphasizes the analogies with algebraic numbers. Unfortunately the partisans of either point of view have what the author describes as "strong emotional reactions". He does not see any virtue in a synthesis, and says, "without attacking in any way the validity per se of the geometric approach, we have not tried to hide our partiality to the algebraic attitude, which has been ours in writing this book". He seems to imply that the geometric and the algebraic methods are the only ones available, but some consider that a third method, that of the theory of functions, used by Riemann himself, is still in many ways the most powerful of all.

Chapter 1 is entitled "Places and Divisors". Instead of starting with complex numbers, Prof. Chevalley starts with the more general concept of a field. This has the advantage (which is not mentioned) that the greater part of Chapters 1,3 and 4 applies with little change to number-fields. Chapter 2 deals with the genus and the theorem of RiemannRoch, which is proved very briefly by regarding differentials from an unusual point of view (to be proved equivalent to the usual one in a later chapter). Chapter 3 introduces the $p$-adic completions of the function fields. Chapter 4 deals with extensions of fields of algebraic functions of one variable. In Chapter 5, which deals with extensions of fields of constants, some may think that the algebraic method shows its weakness, as it has great difficulty in proving properties that are easily dealt with geometrically. Chapter 6 returns to differentials, which have already been used in some earlier chapters. Some of the questions discussed in Chapters 4, 5 and 6 have never been treated before. Chapter 7 , much longer than any of the other chapters, deals with the Riemann surface, not from the usual point of view (which the author describes as the "scissor and glue" method), but by an abstract method for which the author claims "we have avoided the cumbersome decomposition of the Riemann surface into triangles". This chapter contains some interesting and original features, but whether it is an improvement on the usual treatment is open to doubt. The book concludes with an index and a short bibliography.

The author's treatment, it may be conceded, appears to be the best yet known for the study of certain parts of the subject, and has the merit ot pointing out opportunities for further research. If may therefore be commended to the advanced student, with the proviso that he should certainly seek elsewhere a knowledge of other aspects of the subject, so that he can see the relation of the specialized approach to the general body of mathematics as a whole.

H. T. H. PiagGio

1 "Science and Humanism" (Cambridge University Press).

2 Bull. Amer. Math. Soc., 384 (September 1951). 